\title{
Experimental study on dynamic compressive behaviour of sandwich panel with shear thickening fluid filled pyramidal lattice truss core
}

\author{
Xianqian $\mathrm{Wu}^{\mathrm{a}} \mathrm{b}, \mathrm{e}, *$, Kailu Xiao ${ }^{\mathrm{a}, \mathrm{b}}$, Qiuyun Yin ${ }^{\mathrm{c}, * *}$, Fachun Zhong ${ }^{\mathrm{d}}$, Chenguang Huang ${ }^{\mathrm{a}, \mathrm{b}}$ \\ a Key Laboratory of Mechanics in Fluid Solid Coupling Systems, Institute of Mechanics, Chinese Academy of Sciences, Beijing 100190, China \\ ${ }^{\mathrm{b}}$ School of Engineering Science, University of Chinese Academy of Sciences, Beijing 100049, China \\ ${ }^{\mathrm{c}}$ School of Engineering, Sun Yat-Sen University, Guangzhou 510275, China \\ ${ }^{d}$ Institute of Chemical Materials, China Academy of Engineering Physics, Mianyang 621900, China \\ e State Key Laboratory of Explosion Science and Technology, Beijing Institute of Technology, Beijing 100081, China
}

\section{A R T I C L E I N F O}

\section{Keywords:}

Dynamic compressive behaviour

Sandwich panel with pyramidal lattice truss core

Shear thickening fluid

SHPB

Transformation of deformation modes

\begin{abstract}
A B S T R A C T
The dynamic compressive behaviour of sandwich panels with shear thickening fluid (STF) filled pyramidal lattice truss cores at high strain rates is studied and compared with that of pure STF as well as the sandwich panels with empty and water filled pyramidal lattice truss cores by modified split Hopkinson pressure bar (SHPB) apparatus. The dynamic compressive strengths of the sandwich panels while filled with STF increase significantly when compared to the strengths of the sandwich panels with empty pyramidal lattice truss cores. It is interesting to note that the sandwich panel with the STF filled pyramidal lattice truss core shows " $1+1>>2$ " dynamic energy absorption behaviour. The excellent energy absorption behaviour of the sandwich panel with STF filled truss core is interpreted by the transformation of deformation modes of core beams from non-symmetry to symmetry after filled with the STF through strong interaction between the buckling behaviour of pyramidal lattice truss core and the shear thickening behaviour of the filled STF material.
\end{abstract}

(C) 2018 Elsevier Ltd. All rights reserved.

\section{Introduction}

Sandwich panels with lattice truss cores have attracted a lot of attentions of researchers for the past few years due to its lightweight, high strength/density ratio, as well as convenient fabrication [1-3]. It has founded applications in variety of industrial fields such as automobiles, aircrafts, to name a few [4]. Numerous studies address the mechanical responses of sandwich panels with lattice truss cores [5-13]. Having shown high energy absorption capacity through deformation of panels and buckling behaviour of truss core's beams under impulse loading [14-20], metallic sandwich structures still suffer from some shortcomings. For example, the strength of the sandwich panels with lattice truss cores is still relative low when compared to the monolithic plates. Also, it is very difficult to optimize the design of sandwich panels over a wide range of impulses due to the dramatic variation in the stiffness of a core when subjected to buckling followed by re-strengthening [3,21,22].

It is an effective method to use sandwich panels integrated with some advanced materials to further improve its mechanical behaviour. For instance, the study by Wadley et al. [23] showed that filling the cores of sandwich panels with alumina prisms led to severe projectile plastic de- formation and fragmentation as the impact speed was increased, and the ballistic limit increased significantly from about $530-590 \mathrm{~m} / \mathrm{s}$ to approximately $1300 \mathrm{~m} / \mathrm{s}$. Recently, sandwich structures integrated with shear thickening fluid (STF) attracts great attentions of researchers. After filled with STF, the mechanical behaviour of structures such as vibration and damping properties were significantly modified [24,25].

STF is one kind of smart materials that show reversible energy absorption behaviour under impulse loading [26-28]. In addition, it shows excellent energy absorption capacity through viscos dissipation during the transition from a fluid-like state to a solid-like state, i.e. shear thickening behaviour by shear deformation [27,29-31] and compression thickening behaviour by compression [26, 27,32-34]. More important, the shear thickening behaviour is observed to be a result of particles rearrangement from an ordered state to a disordered state, e.g. formation of jamming particle clusters during shear loading [27,31], and the compression thickening behaviour is observed to be a result of impact induced solidification [27,32,33]. When the impulse loading is removed, the viscosity of STF recovers rapidly to the initial state [31], showing the reversible energy absorption capacity.

Combining STF and sandwich panel with lattice truss core can bring a promising structural material with excellent multifunction. It has

* Corresponding author at: Key Laboratory of Mechanics in Fluid Solid Coupling Systems, Institute of Mechanics, Chinese Academy of Sciences, Beijing 100190, China.

** Corresponding author.

E-mail addresses: wuxianqian@imech.ac.cn (X. Wu), yinqy5@mail.sysu.edu.cn (Q. Yin). 
not only the merits of sandwich panel with lattice truss core such as lightweight, high strength/density ratio $[7,10]$, but also the unique properties of STF such as convenient filling, reverse energy absorption behaviour [26,32,33]. More important, since the viscosity of STF is dependent to the impulse condition, it provides a good method to optimize the design of the sandwich panels over a wide range of impulses by controlling the buckling and post buckling behaviour of lattice truss core beams through strong fluid-structure interaction (FSI).

In this paper, the dynamic behaviour of sandwich panels with empty, water filled, and STF filled pyramidal lattice truss cores at various impact velocities are measured by the modified SHPB. After filled with the STF, the energy absorption capacity of the sandwich panel is significantly improved and shows " $1+1>>2$ " phenomenon by transforming the deformation modes from non-symmetrical buckling to symmetrical buckling of core beams.

The paper is organized as follows. In Section 2, the experimental procedure used for preparing the sandwich panels with empty, water filled, and STF filled pyramidal lattice truss cores and the extension of the split Hopkinson pressure bar method for dynamic behaviour testing on large size sandwich panels are described in detail. In Section 3, the experimental conditions and corresponding experimental results are provided. In Section 4, experimental results on pure STF specimens and sandwich panels with empty, water filled, and STF filled pyramidal lattice truss cores at various strain rates are compared, and discussion relevant to the experimental results is provided.

\section{Material fabrication and experimental method}

\subsection{Sandwich panels}

The pyramidal lattice truss cores are harvested through folding $60^{\circ}$ along the short diagonals of a $0.7 \mathrm{~mm}$ in thickness and $12 \mathrm{~mm}$ in length rhombic (acute angles $60^{\circ}$ ) perforated stainless steel sheet, leading to unite truss cells with $7.0 \mathrm{~mm}$ in thickness, $16 \mathrm{~mm}$ in length, and $9 \mathrm{~mm}$ in width. To improve the welding quality between truss cores and panels, $3 \mathrm{~mm}$ width flat areas at nodal regions of the pyramidal lattice truss cores are fabricated, obtaining a relative density $\bar{\rho}$ of about $2.3 \%$ [35]. Due to the folding process the core beams are a little bent at the welding position. The brazing technique with solder material $\mathrm{BNi}-2$ that heats up to $1040-1060^{\circ} \mathrm{C}$ and holds for $10-15 \mathrm{~min}$ is undertaken to weld the pyramidal lattice truss cores and 0.9 -mm-thick stainless steel panels. The detail for preparing the sandwich panel with pyramidal lattice truss core is given by the study of Yuan et al. [36]. The schematic of a typical sandwich panel specimen with three unit cells in length (about $48 \mathrm{~mm}$ ) and five unit cells in width (about $45 \mathrm{~mm}$ ) for dynamic compression is shown in Fig. 1. While filling water or STF, a strip of 0.1-mm-thick soft rubber with an elastic modulus of about $0.5 \mathrm{MPa}$ is used to seal all open sides of a sandwich panel with pyramidal lattice truss core to make a simple mode. For pure STF test, the STF with pre-determined volume is filled into a pocket that made from the same rubber, and then it is sandwiched in experiments to obtain a disk shaped STF specimen for dynamic compression.

\subsection{STF specimens}

The colloidal nanoparticles employed here are monodispersed silica microspheres, which were prepared by the method of Stöber [37]. Briefly, the mixture solution of TEOS and absolute ethanol are slowly introduced into the mixture of ammonia and absolute ethanol by dripping. The silica microspheres are centrifuged, rinsed by ethanol, and finally dried in a vacuum oven at $80{ }^{\circ} \mathrm{C}$ prior to use. The STF are prepared as follows: the silica microspheres, polyethylene glycol 200 (PEG 200 ) and absolute ethanol is added into a round flask. The weight ratio of the silica microspheres and PEG 200 is 70/30, and absolute ethanol, which is used as solvent, is about three times the weight of the silica microspheres and PEG 200. The flask is sonicated for more than four

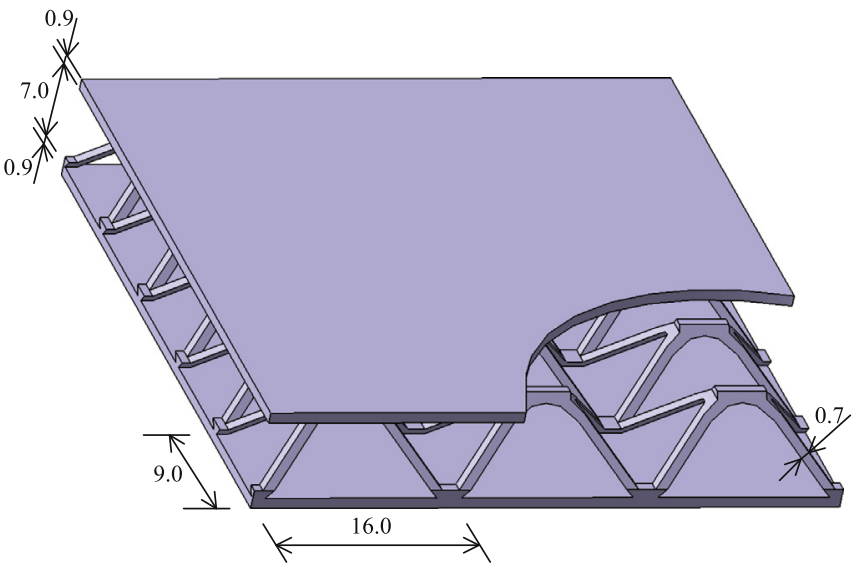

All dimensions in $\mathrm{mm}$

Fig. 1. Schematic of the sandwich panel with pyramidal lattice truss core.

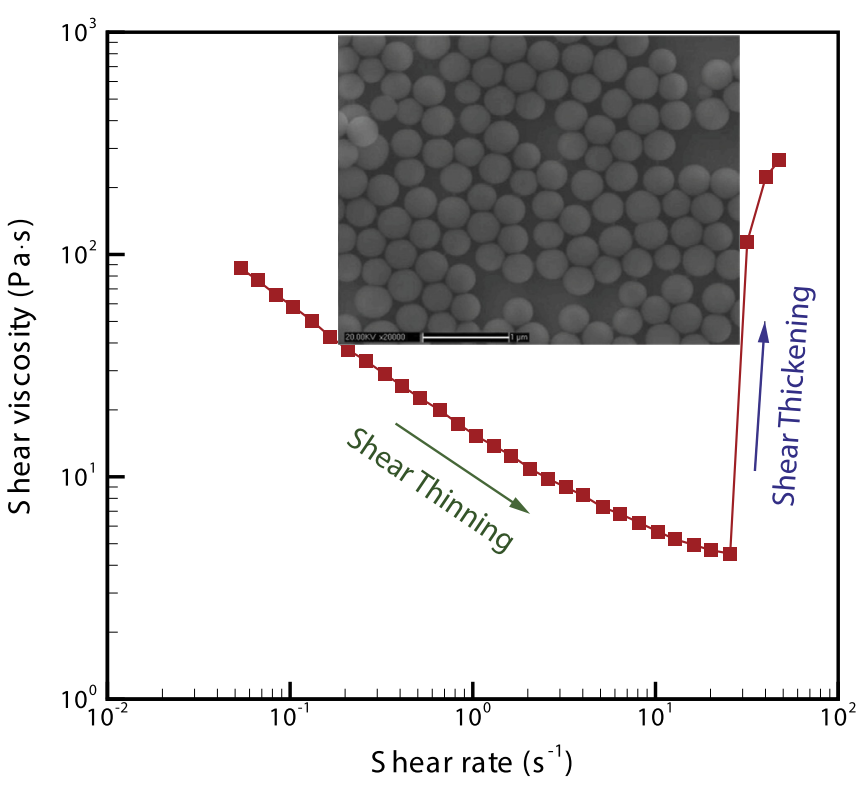

Fig. 2. Steady state shear viscosities of the STF at various strain rates. The decrease and quick increase of shear viscosities of the STF correspond to the shear thinning and the shear thickening regime, respectively. The insert shows that the nearly monodispersed silica sphere particles with an average diameter of $300 \mathrm{~nm}$ with less than $10 \%$ polydispersity.

hours to ensure the complete dispersion of the silica microspheres. Finally, the STF is obtained by rotary evaporated the ethanol from the mixed solution, and sealed in a vial before use.

The STF studied in this paper has a particle volume fraction of $57 \%$. As shown in Fig. 2, the silica sphere particles are nearly monodisperse with an average diameter of $300 \mathrm{~nm}$ with less than $10 \%$ polydispersity. The densities of the silica particles and the polyethylene glycol are $1.950 \mathrm{~g} / \mathrm{cm}^{3}$ and $1.127 \mathrm{~g} / \mathrm{cm}^{3}$, respectively. The sound velocity of the silica particles is $5869 \mathrm{~m} / \mathrm{s}$. The bulk modulus of the polyethylene glycol is $3.05 \mathrm{GPa}$, yielding a sound velocity of $1650 \mathrm{~m} / \mathrm{s}$. The steady state shear viscosity of the STF at various shear strain rates, which are measured by a Kinexus pro +rotational rheometer in the steady-state shear sweep mode using a $40 \mathrm{~mm} 4^{\circ}$ cone and plate with Peltier temperature control at $25{ }^{\circ} \mathrm{C}$, is also plotted in Fig. 2. The STF exhibits a shear thinning regime, followed by a strong shear thickening response at a shear rate of about $25 \mathrm{~s}^{-1}$ as evident by the sharp increase of the shear viscosity. Due to the limitation of the rotational rheometer, the viscosities of the STF at even high shear rates are unable to be obtained. 

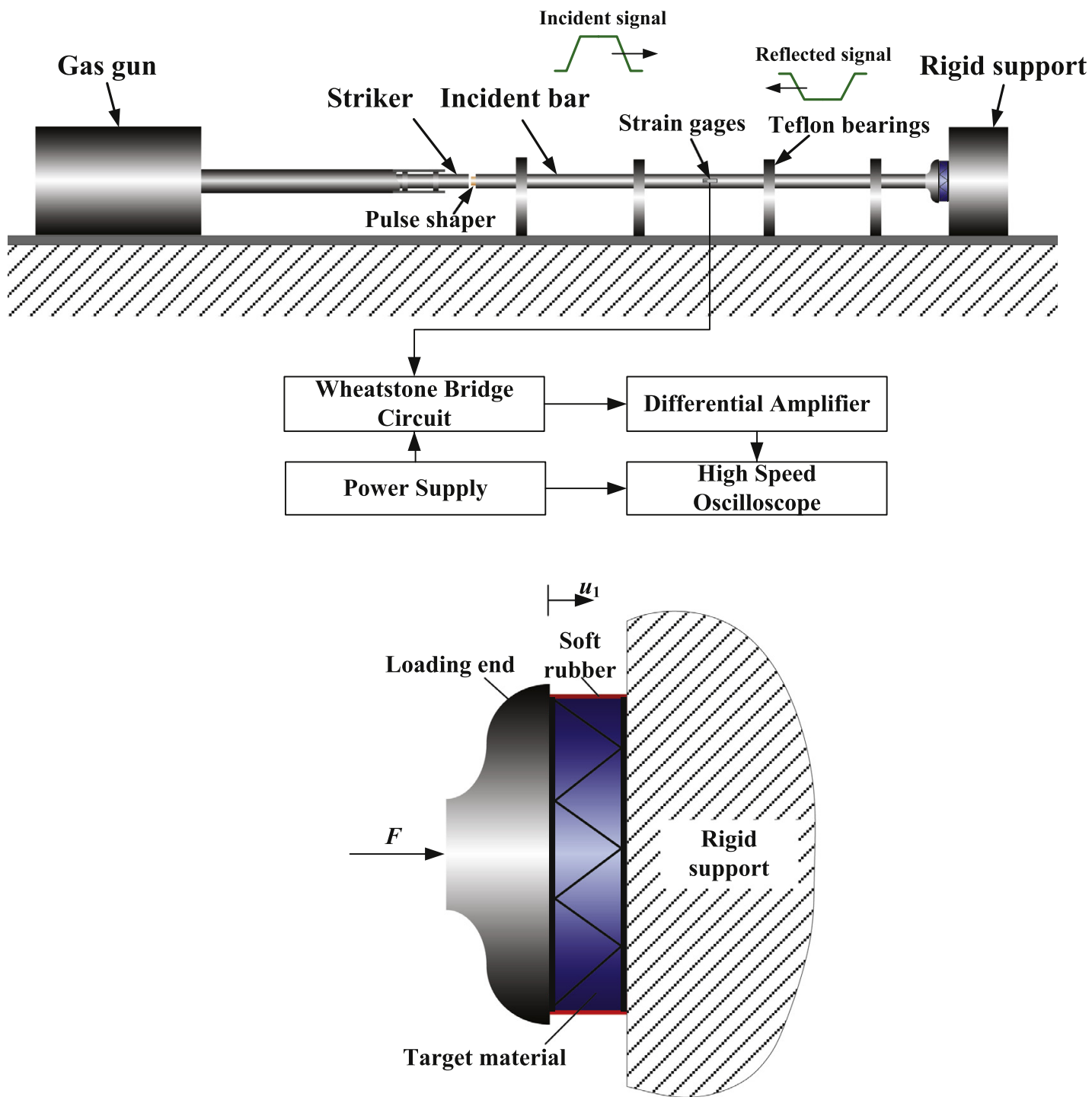

Fig. 3. Modified SHPB apparatus. A trumpet-shaped loading end with a thickness of $15 \mathrm{~mm}$ and a maximum diameter of $80 \mathrm{~mm}$ is employed to compress the sandwich panels with relative large section areas.

\subsection{Experimental method}

In the present study, SHPB apparatus $[38,39]$ is modified to investigate dynamic behaviour of sandwich panels with empty, water filled, and STF filled pyramidal lattice truss cores at nominal strain rates varying from 300 to $1700 \mathrm{~s}^{-1}$. The schematic of the modified SHPB is shown in Fig. 3. The facility comprises a striker bar and an incident bar, all made from $19.05 \mathrm{~mm}$ diameter high-strength 7075-T6 aluminum alloy having a nominal yield strength of approximately $500 \mathrm{MPa}$. Since relative large sizes of sandwich panels are taken in experiments when compared to the diameter of the incident bar to minimize the size effect of the sandwich panels, a trumpet-shaped loading end with a thickness of $15 \mathrm{~mm}$ and a maximum diameter of $80 \mathrm{~mm}$ is employed. The trumpet-shaped end is also made from 7075-T6 aluminum alloy to minimize the impedance mismatch effect between the incident bar and the loading end. The sandwich panel specimens are sandwiched between the trumpet-shaped loading end and a large hard steel disk fabricated from Carpenter Hampden $(\mathrm{CH})$ tool-steel that acts as a rigid support. The tool-steel disk is $150 \mathrm{~mm}$ in diameter and $100 \mathrm{~mm}$ in thickness. The length of the striker bar and the incident bar are approximately $0.7 \mathrm{~m}$ and $1.8 \mathrm{~m}$, respectively. The striker bar is accelerated using a compressed air gas-gun to impact velocities in the range of 5 to $16 \mathrm{~m} / \mathrm{s}$; corresponding to nominal strain rates $300-1700 \mathrm{~s}^{-1}$ in the sandwich panel specimens, respectively. A pair of semiconductor strain gages (SB5-120-P-2Y15) strategically attached on the middle of the incident bar is utilized in combination with a Wheatstone bridge circuit, a differential amplifier, and a digital oscilloscope (LeCroy WaveRunner $620 \mathrm{Zi}$ ) to record the strain pulses in the incident bar during the dynamic tests.

In a SHPB test with the aforementioned configuration, a compressed air gas-gun is used to accelerate the striker bar to impact the incident bar at a pre-determined velocity. The impact results in an elastic compression wave with a strain profile, denoted by $\varepsilon_{I}(t)$, which travels in the incident bar towards the sandwich panel specimen. Due to the impedance mismatch between the specimen and the pressure bars, part of the incident compressive wave is reflected back into the incident bar, denoted by $\varepsilon_{R}(t)$, while the rest is transmitted into the large hard tool-steel disk. Since the impedance of the hard tool-steel disk is much large when compared to the sandwich panel specimen, the tool-steel disk is regarded as a rigid support, and correspondingly the contacted end surface of the sandwich panel specimen is regarded as stationary. Under the assumption of homogeneous deformation, elementary 1D elastic wavepropagation equations are used to calculate the engineering stress $\sigma_{S}(t)$, the strain rate $\dot{\varepsilon}_{S}(t)$, and strain $\varepsilon_{S}(t)$ in the sandwich panel specimen as 


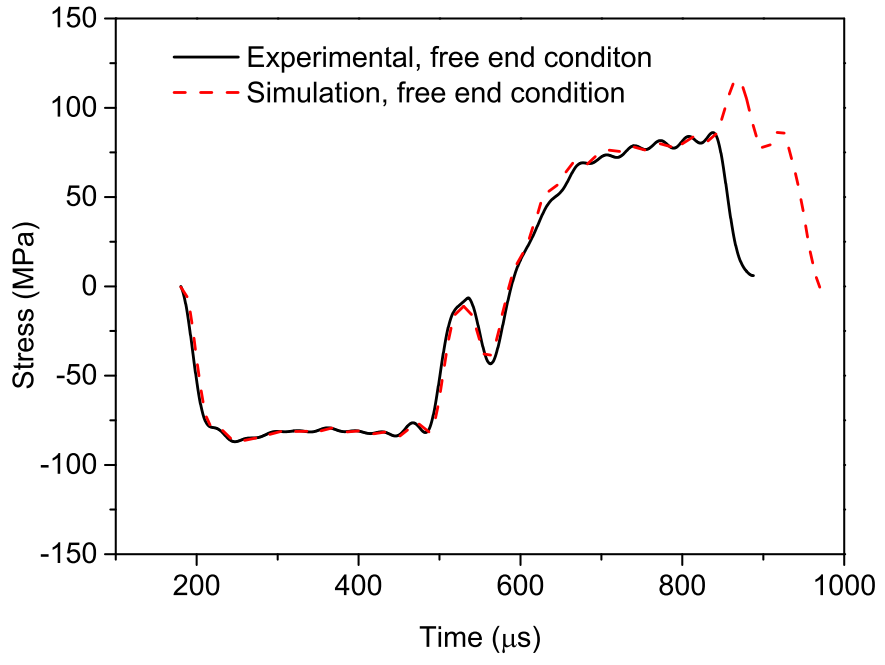

Fig. 4. Validation of the numerical model for simulating the stress pulses in the incident bar with the trumpet-shaped loading end.

$\sigma_{S}(t)=E \frac{A_{0}}{A_{S}}\left[\varepsilon_{I}(t)+\varepsilon_{R}(t)\right]$

$\dot{\varepsilon}_{S}(t)=-2 \frac{c_{0}}{h_{S}} \varepsilon_{R}(t)$

$\varepsilon_{S}(t)=\int_{0}^{t} \dot{\varepsilon}_{S}(t) d t$.

In Eqs. (1)-(3), $E, A_{0}$ and $c_{0}$ are Young's modulus, cross-sectional area, and longitudinal wave speed in the incident bar; $A_{\mathrm{S}}$ and $h_{\mathrm{S}}$ are the initial cross-sectional area and thickness of the specimen, respectively. The true stress-strain relationship is determined from the engineering stress-strain relationship by assuming uniform and isochoric deformation conditions to prevail within the specimen during the deformation process.

\subsection{Data reduction method}

In the experiments a trumpet-shaped loading end with a thickness of $15 \mathrm{~mm}$ and a maximum diameter of $80 \mathrm{~mm}$ is employed to pressure the large size sandwich panel specimens. Although the same7075-T6 aluminum alloy is used for the trumpet-shaped end as the incident bar, the impedance mismatch between the incident bar and the trumpet-shaped loading end is also observed in experiments due to the irregular geometry of the trumpet-shaped loading end. Therefore, the data reduction method with the aid of numerical simulation is implemented to remove the errors introduced by the impedance mismatch between the incident bar and the loading end. The detail of the method is as follows. For each test with a sandwich panel specimen, the incident wave and the reflected wave in the incident bar are measured. Then, the LS-DYNA explicit package [40], which has been validated for simulating SHPB problem [41], is taken to analyze the reflected wave in the incident bar without the sandwich panel specimen, i.e. the free end condition. Only the incident bar and the trumpet-shaped end, which are supposed to be an integral rod, are simulated. The measured incident stress wave, as the loading condition, is applied on the left end surface of the incident bar. The measured stress wave and the simulated stress waves in the middle of the incident bar are given in Fig. 4. It can be seen that the simulated stress wave agrees well with the measured stress wave before $840 \mu \mathrm{s}$. After this time the measured reflected wave begins to unloading whereas the simulated reflected wave continues to loading,

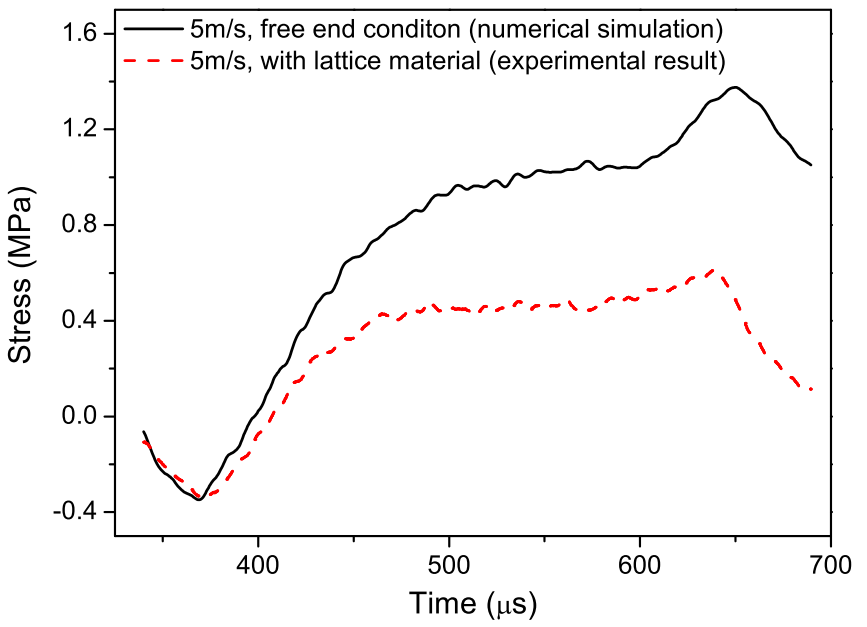

Fig. 5. Typical measured reflected wave (with sandwich specimen) and numerical reflected wave (without sandwich specimen). The same value at the beginning indicates the impedance mismatch between the incident bar and the trumpet-shaped end.

which should be ascribed to the boundary condition between the incident bar and the trumpet-shaped loading end. As the incident bar just contacts other than integrate with the loading end in experiments, the incident bar and the trumpet-shaped loading end will separate when the unloading wave in the incident bar arrives at the trumpet-shaped loading end. As a result, only partial reflected stress wave can be measured in the experiments. However, it is adequate to measure the dynamic compressive behaviour of the sandwich panels. A typical measured reflected wave while compressing a sandwich panel with pyramidal lattice truss core and the simulated reflected wave with free end condition are given in Fig. 5. As shown in Fig. 5, at the beginning the simulated reflected stress wave is almost the same as the measured reflected stress wave, indicating that the reflected wave in this duration is generated by the impedance mismatch between the incident bar and the trumpet-shaped end. Then the measured reflected wave is lower than the simulated reflected wave, showing the actual loading on the sandwich panel specimen. For each test the simulated stress wave minus the measured stress wave gives the correct stress applied on the specimen.

\section{Dynamic compressive results}

The experiments are performed on sandwich panels with empty pyramidal lattice truss cores (SPEC), water filled pyramidal lattice truss core (SPWC), STF filled pyramidal lattice truss cores (SPSC), and pure STF (STF) at various impact velocities. The experimental parameters of all the tests are summarized in Table 1 . Due to the nonuniform deformation of the pyramidal lattice truss cores during fabrication, the sizes of unit cells have slight difference.

\subsection{Results of sandwich panels with empty pyramidal lattice truss cores}

The nominal stress versus strain relationships of the sandwich panels with empty pyramidal lattice truss cores at impact velocities of about $5 \mathrm{~m} / \mathrm{s}$ and $10 \mathrm{~m} / \mathrm{s}$, corresponding to strain rates of approximately $420 \mathrm{~s}^{-1}$ and $900 \mathrm{~s}^{-1}$, respectively, are shown in Fig. 6. The result under quasi-static compression is also given in the figure as a comparison. At the beginning the stresses increase almost linearly with the increase of strain. The maximum elastic strain is 0.058 and 0.078 for quasi-static compression and dynamic compression, respectively. Then the buckling behaviour of the pyramidal lattice trusses happens, leading to the decreases of stresses with further compression. While the pyramidal lattice truss contact with each other or with the panels, the stresses increase 
Table 1

Experimental parameters for experiments involving compression on various specimens.

\begin{tabular}{llllll}
\hline Test no. & Test specimen & Specimen size $(\mathrm{mm})$ & Unit cells & Impact velocity $(\mathrm{m} / \mathrm{s})$ & Strain rate $\left(\mathrm{s}^{-1}\right)$ \\
\hline 1 & SPEC-1 & $100.13 \times 100.08$ & $6 \times 11$ & $3 \times 10^{-5}$ & 0.004 \\
2 & SPEC-2 & $46.18 \times 36.14$ & $3 \times 4$ & 5 & 420 \\
3 & SPEC-3 & $48.20 \times 36.38$ & $3 \times 4$ & 10 & 900 \\
4 & STF-1 & $\Phi 52.80$ & - & 5 & 540 \\
5 & STF-2 & $\Phi 52.73$ & - & 8 & 920 \\
6 & STF-3 & $\Phi 52.76$ & - & 13 & 1500 \\
7 & SPWC-1 & $49.08 \times 37.05$ & $3 \times 4$ & 16 & 1400 \\
8 & SPSC-1 & $46.84 \times 37.10$ & $3 \times 4$ & 5 & 430 \\
9 & SPSC-2 & $49.18 \times 36.88$ & $3 \times 4$ & 10 & 840 \\
10 & SPSC-3 & $46.20 \times 37.00$ & $3 \times 4$ & 16 & 1300 \\
\hline
\end{tabular}

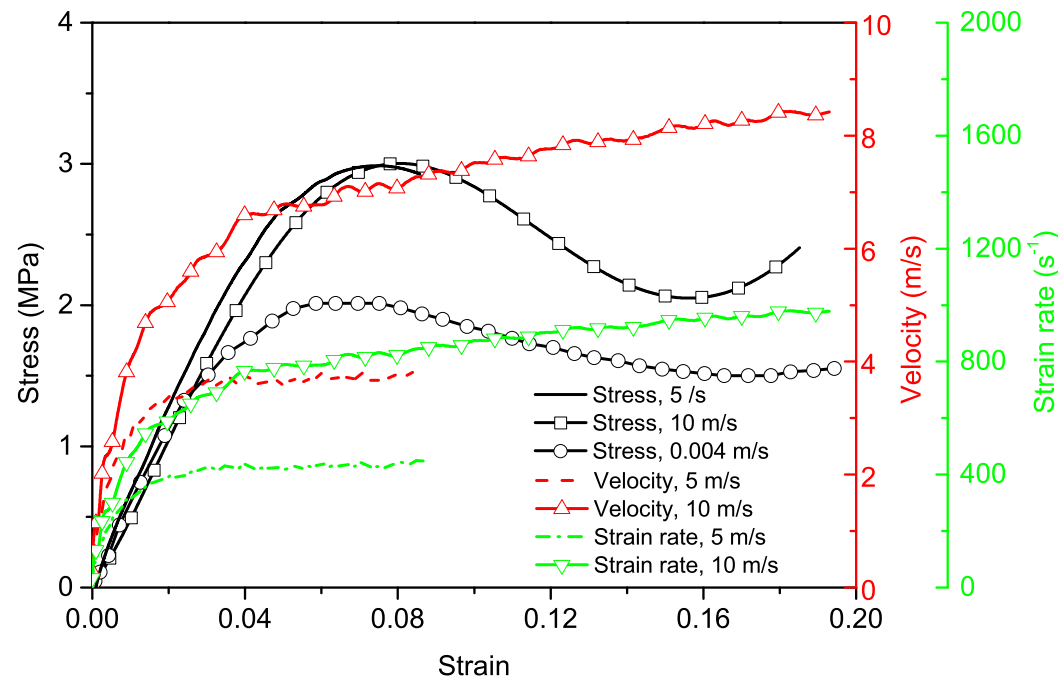

Fig. 6. Nominal stress versus strain relationships of the sandwich panels with empty pyramidal lattice truss cores at various strain rates.

quickly once again with increasing strain. Since the strain rates are almost at the same level, the results obtained at impact velocities $5 \mathrm{~m} / \mathrm{s}$ and $10 \mathrm{~m} / \mathrm{s}$ is not obvious. The strength of the sandwich panel obtained at quasi-static compression is about $2.0 \mathrm{MPa}$, while the strength is approximately 3.0 MPa at a strain rate of about $900 \mathrm{~s}^{-1}$, which is about $50 \%$ higher than that obtained at quasi-static compression due to the lateral effects as the studies by Lee et al. [42], Tang et al. [43], and Vaughn et al. [44].

\subsection{Results of pure STF specimens}

The compressive behaviour of the pure STF at impact velocities of about $5 \mathrm{~m} / \mathrm{s}, 8 \mathrm{~m} / \mathrm{s}$ and $13 \mathrm{~m} / \mathrm{s}$, corresponding to strain rates of about $540 \mathrm{~s}^{-1}, 920 \mathrm{~s}^{-1}, 1500 \mathrm{~s}^{-1}$, are shown in Fig. 7. For the impact velocities of $5 \mathrm{~m} / \mathrm{s}$ and $8 \mathrm{~m} / \mathrm{s}$, at the beginning the stresses increase quickly with the increases of strain, and then it keeps at plateaus of about $0.5 \mathrm{MPa}$ and 1.2 MPa, respectively, with a short period and increase again with continuous loading. At the impact velocity of $13 \mathrm{~m} / \mathrm{s}$, the compressive resistance of the STF continues to increases, and the stress plateau as observed at the impact velocities of $5 \mathrm{~m} / \mathrm{s}$ and $8 \mathrm{~m} / \mathrm{s}$ is not distinct; instead the stress increases slightly with increasing strain.

\subsection{Result of sandwich panel with water filled pyramidal lattice truss core}

The nominal stress versus strain relationship of the sandwich panels with distilled water filled pyramidal lattice truss core at an impact velocity of about $16 \mathrm{~m} / \mathrm{s}$, corresponding to a strain rate of about $1400 \mathrm{~s}^{-1}$, is shown in Fig. 8. The stress of the sandwich increases quickly to the peak value of approximately 4.1 MPa at the strain 0.14 . With continu- ous compression the stress decreases to a value of about 3.0 MPa at the strain 0.26 due to the buckling and post buckling behaviour of the pyramidal lattice truss. After that the stress increases quickly with increasing strain while the pyramidal lattice truss contact with each other or with the panel. According to the measurement results as shown in Fig. 6, the strengths of the sandwich panels with empty pyramidal lattice truss core are almost the same in the strain rate range $420-900 \mathrm{~s}^{-1}$. Regarding the strength of the empty sandwich at strain rate $1400 \mathrm{~s}^{-1}$ is the same as obtained at strain rate $900 \mathrm{~s}^{-1}$ because the strain rates are at the same level, the peak stress of the sandwich panel with water filled pyramidal lattice truss core increases by about $36.7 \%$ due to the inertial effect of the filled water when compared to the strengths of the sandwich panels with empty pyramidal lattice truss cores.

\subsection{Results of sandwich panels with STF filled pyramidal lattice truss cores}

The nominal stress versus strain relationships of the sandwich panels with STF filled pyramidal lattice truss cores at impact velocities of $5 \mathrm{~m} / \mathrm{s}$, $10 \mathrm{~m} / \mathrm{s}$ and $16 \mathrm{~m} / \mathrm{s}$, corresponding to strain rates of $430 \mathrm{~s}^{-1}, 840 \mathrm{~s}^{-1}$ and $1300 \mathrm{~s}^{-1}$, respectively, are shown in Fig. 9. Due to the limitation of the loading pulse durations for the impact velocities of $5 \mathrm{~m} / \mathrm{s}$ and $10 \mathrm{~m} / \mathrm{s}$, the tendencies of the stresses with increasing strain are not clear when the loading strain exceeds 0.18 . Here, we speculate the stress versus strain curves at impact velocities of $5 \mathrm{~m} / \mathrm{s}$ and $10 \mathrm{~m} / \mathrm{s}$ are the same as that obtained at the impact velocity of $16 \mathrm{~m} / \mathrm{s}$. It can be seen that with the increases of strain to about 0.17 the stresses increase almost linearly to about $9.0 \mathrm{MPa}$, which increases by about 3 times when compared to the dynamic strengths of the sandwich panels with empty pyramidal lattice truss cores, showing the significantly improved dynamic energy 


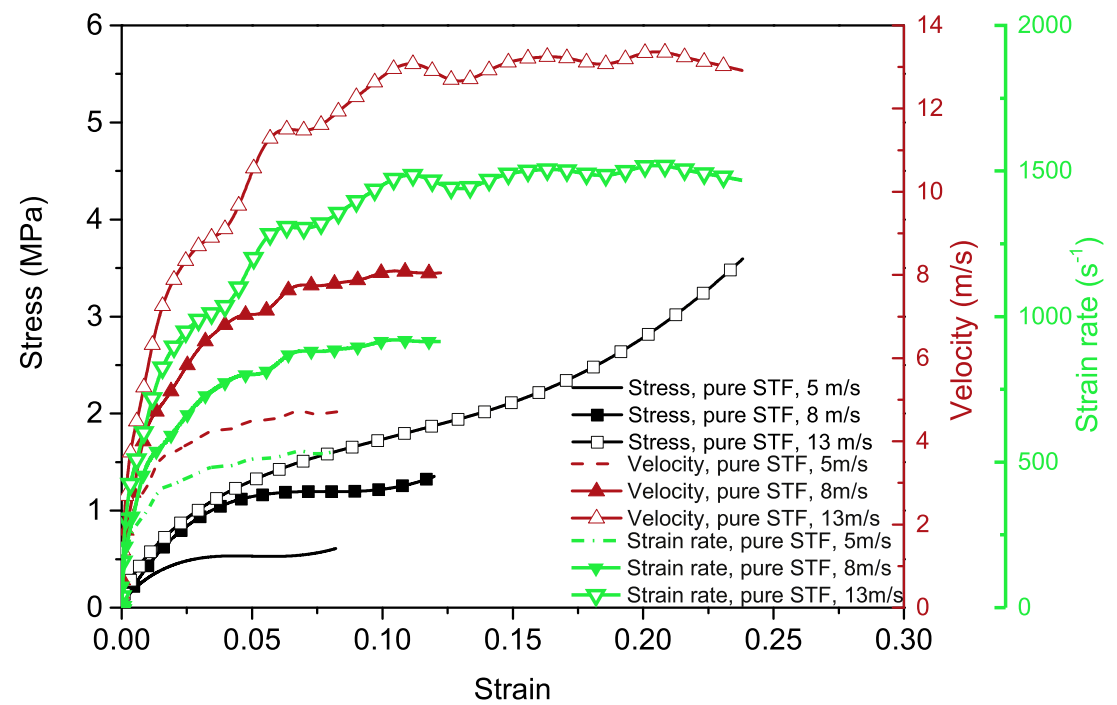

Fig. 7. Nominal stress versus strain relationships of the pure STF specimens at various strain rates.

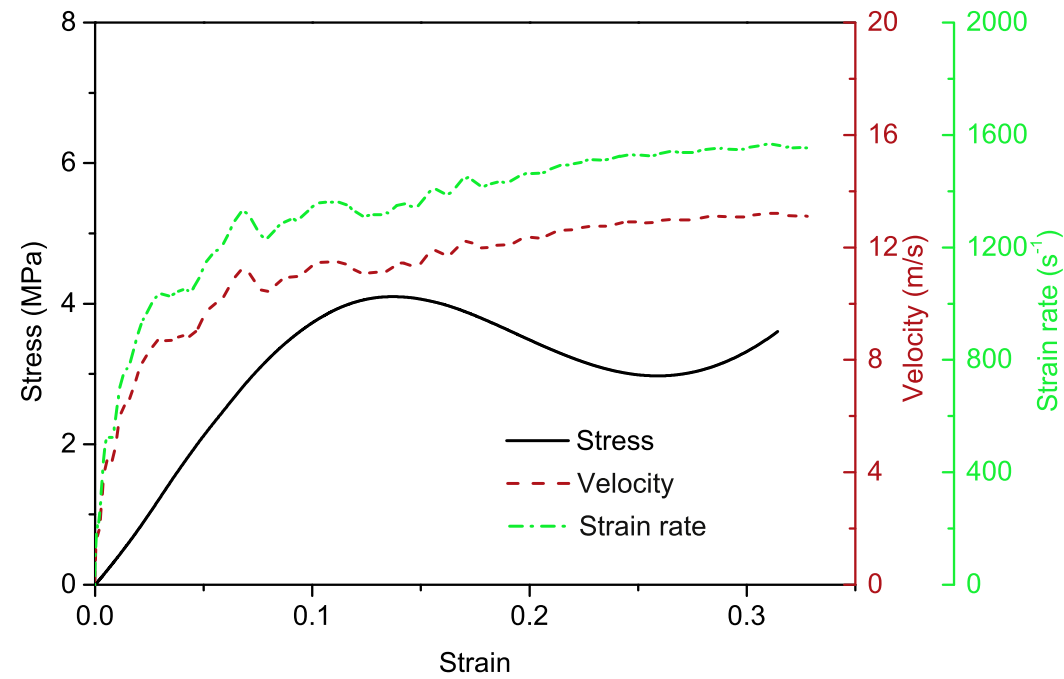

Fig. 8. Nominal stress versus strain relationship of the sandwich panel with water filled pyramidal lattice truss core at a strain rate of about $1400 \mathrm{~s}^{-1}$.

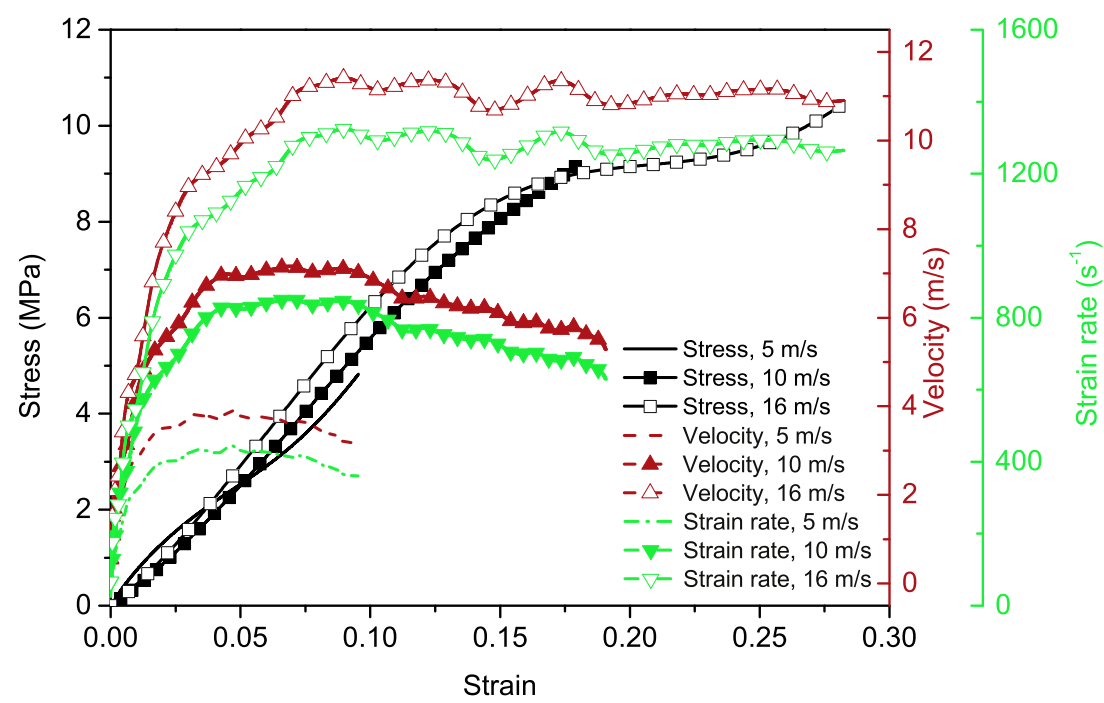

Fig. 9. Nominal stress versus strain relationships of the sandwich panels with STF filled pyramidal lattice truss cores at various strain rates. 


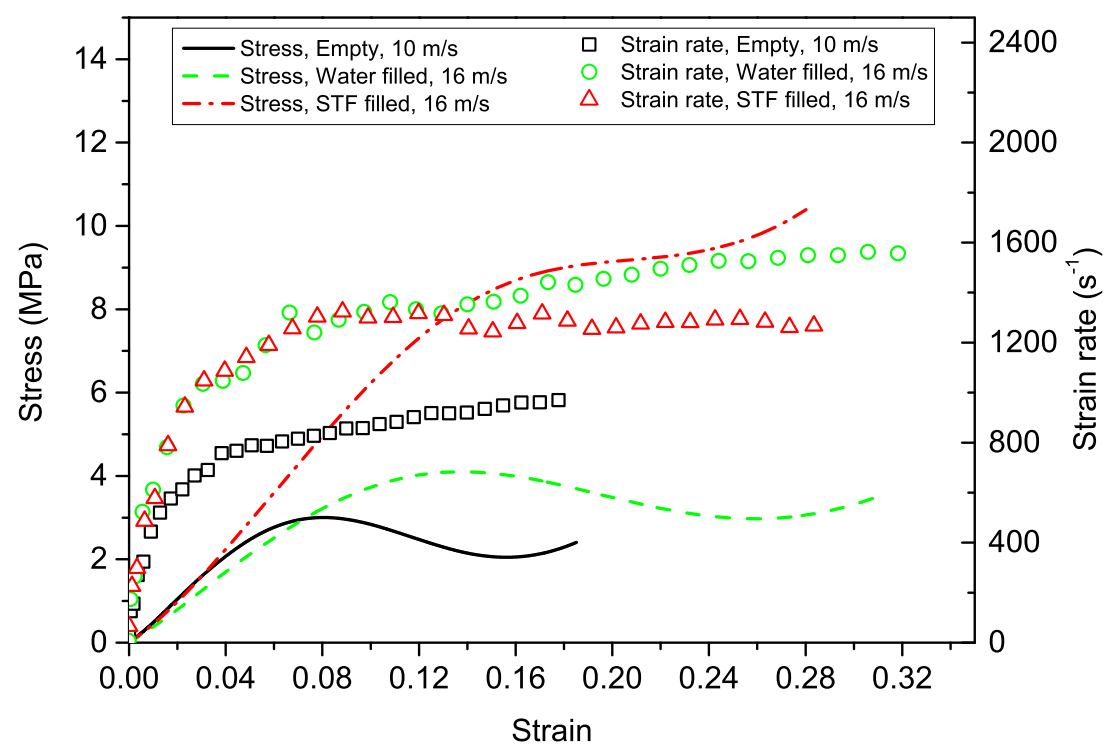

Fig. 10. Nominal dynamic stress versus strain profiles of the sandwich panels with empty, water filled, and STF filled pyramidal lattice truss cores.

absorption capacity of the sandwich panels while filled with the STF. Then the stress increases slowly with further compression, rather than the quick drop of stress induced by the buckling behaviour of empty pyramidal lattice truss cores as shown in Figs. 6 and 8.

\section{Summary of results and discussion}

In the present study the compressive behaviour of pure STF and sandwich panels with empty, water filled, and STF filled pyramidal lattice truss cores are investigated at various strain rates using a modified SHPB. The results show that the strengths of the sandwich panels with empty pyramidal lattice truss cores under quasi-static and dynamic compressions are controlled by the buckling behaviour of the pyramidal lattice trusses, and the dynamic strength of the sandwich panel is higher than that obtained at quasi-static compression condition due to the lateral inertial effect of the pyramidal lattice truss as the studies by Lee et al. [42], Tang et al. [43], and Vaughn et al. [44]. It is interesting to note that while filling fluids into the spaces of pyramidal lattice truss cores, the strength of sandwich panel is improved. The nominal dynamic stress versus strain profiles of the sandwich panels with empty, water filled, and STF filled pyramidal lattice truss cores are compared in Fig. 10. After filled with distilled water the strength of the sandwich panel increases from about 3.0 MPa to about 4.0 MPa, which should be ascribed to the inertial effect of the filled water during compression. Also, the stress decreases slowly after the attainment of the peak stress because of the post buckling behaviour of the pyramidal lattice truss cores, which is similar with the compressive behaviour of the sandwich panels with empty pyramidal truss cores. However, the stresses of the sandwich panel with STF filled pyramidal lattice truss cores increase almost linearly to as high as $9.0 \mathrm{MPa}$, which is almost three times higher when compared to the strengths of the sandwich panels with empty pyramidal lattice truss cores, showing the strong interaction between the pyramidal lattice truss core and the filled STF specimen. Then after, the stress increases slightly with further compression, rather than the slowly decrease as observed for the sandwich panels with empty pyramidal lattice truss cores.

The nominal stress versus strain profiles of the pure STF specimens and the sandwich panels with empty and STF filled pyramidal truss cores are given in Fig. 11. As a comparison, the stress versus strain curve through adding the stresses of pure STF and the sandwich panel with empty truss core is also depicted. It can be seen that while filled with STF, the dynamic energy absorption capacity of the sandwich panel is

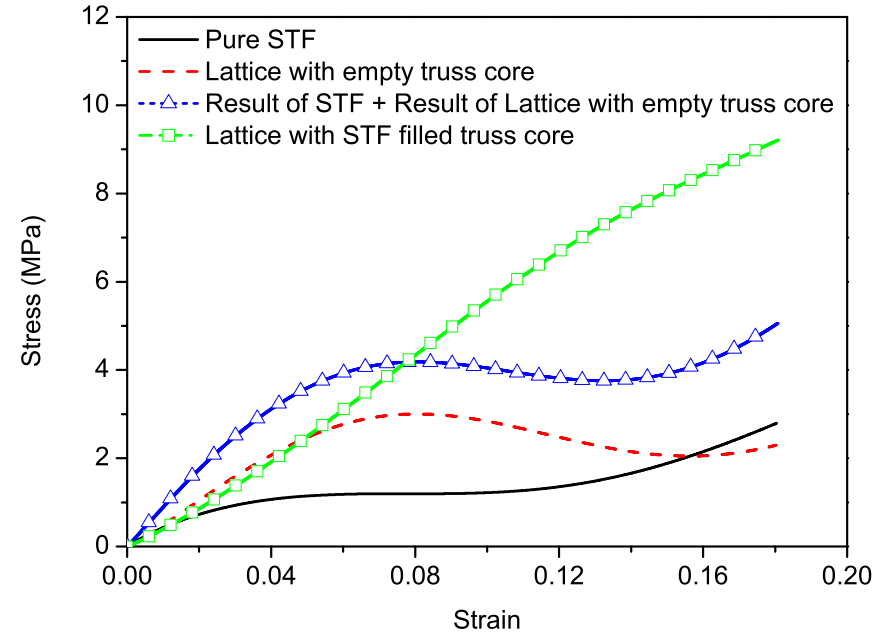

Fig. 11. Nominal stress versus strain profiles of the pure STF specimens and the sandwich panels with empty and STF filled pyramidal truss cores. The stress versus strain curve through adding the stresses of the pure STF and the sandwich panel with empty truss core is also depicted as a comparison.

improved significantly. The stress of the sandwich panel with the STF filled lattice truss core is almost three times higher than the strength of the sandwich panel with empty pyramidal lattice truss core, showing the strong interaction between the truss core and the filled STF specimen. It is interesting to note that the strength of the sandwich panel with the STF filled pyramidal lattice truss core is also much higher than the adding result of pure STF and the sandwich panel with empty truss core, showing the " $1+1>>2$ " dynamic energy absorption behaviour of the sandwich panel while filled with the STF.

The excellent dynamic energy absorption behaviour of the sandwich panel with the STF filled pyramidal lattice truss core could be well understood by the interaction between the filled STF and the pyramidal lattice core. Fig. 12(a) and (b) shows the deformation behaviour of sandwich panels with empty and STF filled pyramidal lattice truss cores after compression, respectively. As shown in Fig. 12(a), during compression the sandwich panel with empty pyramidal lattice truss core, the unit cells of the core experience elastic deformation, leading to the initial fast increase of stress. Then only one side of beams, here is the left 


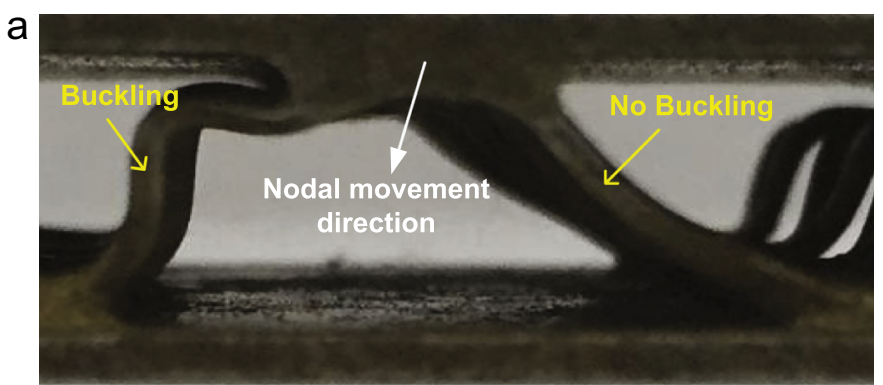

b

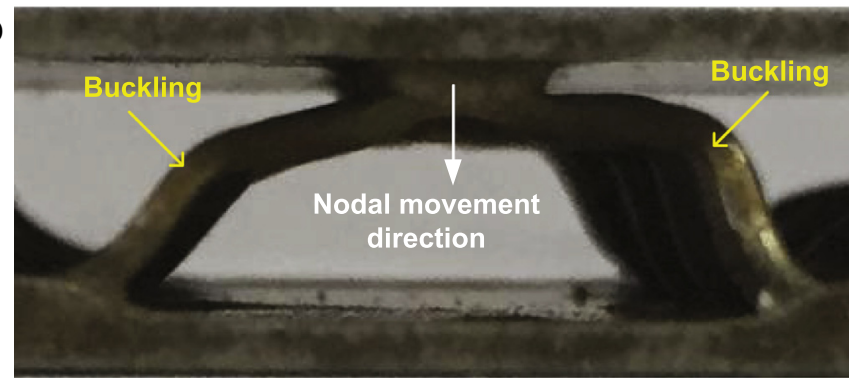

Fig. 12. Deformation behaviour of sandwich panels with (a) empty and (b) STF filled pyramidal lattice truss cores after dynamic compression. Non-symmetrical to symmetrical buckling transition of unit cells happens after filled with the STF.

side, buckle, leading to the attainment of the peak stress. After that the buckled beams experience post-buckling behaviour as evident by the large plastic deformation as shown in Fig. 12(a), resulting in the slow decrease of stress after the attainment of the peak stress. With further compression after the nonsymmetrical buckling of the unit cells, the upper panel moves with a slight obliquity to the normal direction. The unbuckled beams begin to rotate around the bottom welding points that act as hinges, and no buckling behaviour for these unbuckled beams happens in the following compression. However, the deformation modes of the unit cells are changed through strong interaction between the pyramidal lattice truss core and the filled STF material. As shown in Fig. 12(b), once the non-symmetrical buckling behaviour of the unit cells happens, the lateral velocities of the buckled beams are extremely fast when compared to the loading velocity. Consequently, the viscosity of the STF around the beams increases significantly, leading to extremely large drag force that applied on the lateral surface and significant increase of post-buckling force of the buckled beams. Therefore, the unbuckled beams will begin to buckle with continuous compression, resulting in the symmetrical buckling behaviour of the pyramidal lattice truss core as shown in Fig. 12(b). Consequently, the buckling and post-buckling stresses increase significantly for the sandwich panel with STF filled pyramidal lattice truss core, showing the self-reinforced dynamic behaviour through non-symmetrical to symmetrical buckling transition after filled with the STF. Accordingly, excellent dynamic energy absorption behaviour of the sandwich panel with STF filled pyramidal lattice truss core is achieved. More studies will be performed to optimize the design of the combined structural material to further improve its dynamic energy absorption capacity. It is also worth emphasizing that some cracks in STF were observed by thestudy of Waitukaitis and Jaeger [27]. During the buckling and plastic post buckling behaviour of the core beams, the cracks may also generated by the fast lateral movement of the beams, under which condition the resistance of the STF may be controlled not only by the viscosity but also by the fracture strength of the STF. More researches will be conducted to study the dynamic behaviour of theSTF in the shear thickening regime.

\section{Concluding remarks}

The dynamic compressive behaviour of sandwich panels with empty, water filled and STF filled pyramidal lattice truss cores at high strain rates are measured by the modified SHPB experimental. Due to the coupling effect between the lattice truss core beams and the STF, high energy absorption capacity of the sandwich panels with STF filled pyramidal lattice truss cores are obtained and shows " $1+1>>2$ " phenomenon.

(i) The dynamic strengths of sandwich panels with empty and water filled pyramidal lattice truss cores are controlled by the nonsymmetrical buckling and post buckling behaviour of unit cells of lattice truss core. The dynamic strength is about $50 \%$ higher than the static strength due to the lateral inertial effect of the lattice truss during dynamic compression. After filled with water, the dynamic strength is higher than the empty lattice truss cores due to the inertial effect of the filled water.

(ii) The strength at the attainment of the buckling of core beams after filled with the STF is almost three times higher when compared to that of the empty truss cores. Also the stress increases slightly with further compression, rather than the slowly decrease controlled by the non-symmetrical post buckling behaviour of unit cells as observed for the sandwich panels with empty pyramidal lattice truss cores.

(iii) By transforming non-symmetrical buckling behaviour of core beams to symmetrical buckling behaviour and FSI effects after filled with the STF, the sandwich panel with the STF filled pyramidal lattice truss core shows " $1+1>>2$ " dynamic energy absorption behaviour.

\section{Acknowledgements}

The paper was supported by National Natural Science Foundation of China (Grant Nos. 11332011, 11402277, 11472276, 11672315, and 11772347) and the Strategic Priority Research Program of the Chinese Academy of Sciences (Grant Nos. XDB22040302 and XDB22040303).

\section{References}

[1] Gibson LJ, Ashby MF. Cellular solids: Structure and properties. Cambridge University Press; 1999.

[2] Wadley H, Borvik T, Olovsson L, Wetzel J, Dharmasena K, Hopperstad O, Deshpande V, Hutchinson J. Deformation and fracture of impulsively loaded sandwich panels. J Mech Phys Solids 2013;61:674-99.

[3] Hutchinson JW, Xue Z. Metal sandwich plates optimised for pressure impulses. Int J Mech Sci 2005;47:545-69.

[4] Evans AG, Hutchinson J, Ashby M. Multifunctionality of cellular metal systems. Prog Mater Sci 1998;43:171-221.

[5] Deshpande V, Fleck N. Collapse of truss core sandwich beams in 3-point bending. Int J Solids Struct 2001;38:6275-305.

[6] Chiras S, Mumm D, Evans A, Wicks N, Hutchinson J, Dharmasena K, Wadley H, Fichter S. The structural performance of near-optimized truss core panels. Int J Solids Struct 2002;39:4093-115.

[7] Evans AG, Hutchinson JW, Fleck NA, Ashby M, Wadley H. The topological design of multifunctional cellular metals. Prog Mater Sci 2001;46:309-27.

[8] Wallach J, Gibson L. Mechanical behaviour of a three-dimensional truss material. Int J Solids Struct 2001;38:7181-96.

[9] Queheillalt DT, Wadley HN. Cellular metal lattices with hollow trusses. Acta Mater 2005;53:303-13.

[10] Wadley HN. Multifunctional periodic cellular metals. Philos Trans R Soc London A 2006;364:31-68.

[11] Dharmasena KP, Wadley HN, Williams K, Xue Z, Hutchinson JW. Response of metallic pyramidal lattice core sandwich panels to high intensity impulsive loading in air. Int J Impact Eng 2011;38:275-89.

[12] Wei Z, Deshpande V, Evans A, Dharmasena K, Queheillalt D, Wadley H, Murty Y, Elzey R, Dudt P, Chen Y. The resistance of metallic plates to localized impulse. J Mech Phys Solids 2008;56:2074-91.

[13] Xiang XM, Lu G, Wang ZH. Quasi-static bending behaviour of sandwich beams with thin-walled tubes as core. Int J Mech Sci 2015;103:55-62.

[14] Rathbun HJ, Radford DD, Xue Z, He MY, Yang JY, Deshpande VS, Fleck NA, Hutchinson JW, Zok FW, Evans AG. Performance of metallic honeycomb-core sandwich beams under shock loading. Int J Solids Struct 2006;43:1746-63.

[15] Dharmasena KP, Queheillalt DT, Wadley HNG, Dudt P, Chen Y, Knight D, Evans AG, Deshpande VS. Dynamic compression of metallic sandwich structures during planar impulsive loading in water. Eur J Mech A Solids 2010;29:56-67. 
[16] Wei X, Tran P, de Vaucorbeil A, Ramaswamy RB, Latourte F, Espinosa HD. Three-dimensional numerical modeling of composite panels subjected to underwater blast. J Mech Phys Solids 2013;61:1319-36.

[17] Li S, Lu G, Wang Z, Zhao L, Wu G. Finite element simulation of metallic cylindrical sandwich shells with graded aluminum tubular cores subjected to internal blast loading. Int J Mech Sci 2015;96:1-12.

[18] Lim J-Y, Bart-Smith H. High velocity compressive response of metallic corrugated core sandwich columns. Int J Mech Sci 2016;106:78-94.

[19] St-Pierre L, Deshpande VS, Fleck NA. The low velocity impact response of sandwich beams with a corrugated core or a Y-frame core. Int. J Mech Sci 2015;91:71-80.

[20] Xue Z, Hutchinson JW. Preliminary assessment of sandwich plates subject to blast loads. Int J Mech Sci 2003;45:687-705.

[21] Vaziri A, Xue Z, Hutchinson JW. Metal sandwich plates with polymeric foam-filled cores. J Mech Mater Struct 2006;1:95

[22] Mcshane GJ, Deshpande VS, Fleck NA. Underwater blast response of free-standing sandwich plates with metallic lattice cores. Int J Impact Eng 2010;37:1138-49.

[23] Wadley HNG, Dharmasena KP, O'Masta MR, Wetzel JJ. Impact response of aluminum corrugated core sandwich panels. Int J Impact Eng 2013;62:114-28.

[24] Fischer C, Braun S, Bourban P, Michaud V, Plummer C. Dynamic properties of sandwich structures with integrated shear-thickening fluids. Smart Mater Struct 2006;15:1467.

[25] Tan ZH, Zuo L, Li WH, Liu LS, Zhai PC. Dynamic response of symmetrical and asymmetrical sandwich plates with shear thickening fluid core subjected to penetration loading. Mater Des 2016;94:105-10.

[26] Jiang W, Gong X, Xuan S, Jiang W, Ye F, Li X, Liu T. Stress pulse attenuation in shear thickening fluid. Appl Phys Lett 2013;102:101901.

[27] Waitukaitis SR, Jaeger HM. Impact-activated solidification of dense suspensions via dynamic jamming fronts. Nature 2012;487:205-9.

[28] Bender J, Wagner NJ. Reversible shear thickening in monodisperse and bidisperse colloidal dispersions. J Rheol 1996;40:899-916.

[29] Laun H, Bung R, Hess S, Loose W, Hess O, Hahn K, Hädicke E, Hingmann R, Schmidt F, Lindner P. Rheological and small angle neutron scattering investigation of shear-induced particle structures of concentrated polymer dispersions submitted to plane Poiseuille and Couette flowa. J Rheol 1992;36:743-87.

[30] Newstein MC, Wang H, Balsara NP, Lefebvre AA, Shnidman Y, Watanabe H, Osaki K, Shikata T, Niwa H, Morishima Y. Microstructural changes in a colloidal liquid in the shear thinning and shear thickening regimes. J Chem Phys 1999;111:4827-38.
[31] Barnes H. Shear-thickening ("Dilatancy") in suspensions of nonaggregating solid particles dispersed in newtonian liquids. J Rheol 1989;33:329-66.

[32] Wu X, Zhong F, Yin Q, Huang C. Dynamic response of shear thickening fluid under laser induced shock. Appl Phys Lett 2015;106 071903.

[33] Wu X, Yin Q, Huang C. Experimental study on pressure, stress state, and temperature-dependent dynamic behaviour of shear thickening fluid subjected to laser induced shock. J Appl Phys 2015;118:173102.

[34] Lim A, Lopatnikov S, Wagner N, Gillespie J Jr. Investigating the transient response of a shear thickening fluid using the split Hopkinson pressure bar technique. Rheol Acta 2010;49:879-90.

[35] Kooistra GW, Deshpande VS, Wadley HNG. Compressive behaviour of age hardenable tetrahedral lattice truss structures made from aluminium. Acta Mater 2004;52:4229-37.

[36] Yuan W, Song H, Wang X, Huang C. Experimental investigation on thermal buckling behaviour of truss-core sandwich panels. AIAA J 2014;53:948-57.

[37] Stöber W, Fink A, Bohn E. Controlled growth of monodisperse silica spheres in the micron size range. J Colloid Interface Sci 1968;26:62-9.

[38] Kolsky H. An investigation of the mechanical properties of materials at very high rates of loading. Proc R Soc London B 1949;62:676-700.

[39] Hopkinson B. A method of measuring the pressure produced in the detonation of high explosives or by the impact of bullets. Philos Trans R Soc London A 1914;213:437-56.

[40] Hallquist JO. LS-DYNA3D theoretical manual. Livermore Software Technology Corporation; 1993.

[41] Wu X, Yin Q, Wei Y, Huang C. Effects of imperfect experimental conditions on stress waves in SHPB experiments. Acta Mech Sin 2015;31:827-36.

[42] Lee S, Barthelat F, Hutchinson JW, Espinosa HD. Dynamic failure of metallic pyramidal truss core materials - experiments and modeling. Int J Plasticity 2006;22:2118-45

[43] Tang X, Prakash V, Lewandowski JJ, Kooistra GW, Wadley HNG. Inertial stabilization of buckling at high rates of loading and low test temperatures: implications for dynamic crush resistance of aluminum-alloy-based sandwich plates with lattice core. Acta Mater 2007;55:2829-40.

[44] Vaughn DG, Canning J, Hutchinson JW. Coupled plastic wave propagation and column buckling. J Appl Mech 2005;72:139-46. 